\title{
STRICTLY REGULAR ELEMENTS IN FREUDENTHAL TRIPLE SYSTEMS
}

$\mathrm{BY}$

\author{
J. C. FERRAR( $(1)$
}

\begin{abstract}
Strictly regular elements play a role in the structure theory of Freudenthal triple systems analogous to that played by idempotents in nonas sociative algebras with identity. In this paper we study the coordinatization of reduced triple systems relative to a connected pair of strictly regular elements and use the explicit form of strictly regular elements in terms of the coordinatization to prove uniqueness of the coordinatizing Jordan algebra, as well as several generalizations of known results regarding groups of transformations related to triple systems. Finally, we classify forms of a particularly important triple system (the representation module for the Lie algebra $E_{7}$ ) over finite, $p$-adic or real fields.
\end{abstract}

Following Freudenthal [7], Meyberg [9] and Brown [1] have introduced an axiomatic approach to the study of the ternary algebraic structure of the minimal dimensional module for the Lie algebra $E_{7}$. In particular, this module is one of a class of ternary algebras we refer to as Freudenthal Triple Systems (FTS). In this paper we analyze further the internal structure of such algebras using as a basic tool the Peirce decomposition relative to a pair of supplementary strictly regular elements. Using this decomposition we obtain, in a manner similar to [1], a coordinatization theorem-every simple, reduced FTS is isomorphic to $\mathbb{M}(\mathfrak{J}), \mathfrak{J}$ a member of a small class of Jordan algebras specified in $\$ 1$. In $\$ 6$ we analyze the forms of strictly regular elements in $\mathscr{M}(\mathcal{J}$ and use the results to show $M(\Im) \cong M(\Re)$ if and only if $\Im$ and $\Re$ are norm equivalent. In $\$ 7$ the action of the group of $q$-similarities on the strictly regular elements is studied, yielding conjugacy theorems generalizing a result of Brown [1], as well as information regarding ratios of similitudes generalizing results of Faulkner [4] and Seligman (unpublished). Results of this section are useful also in the classification problem for Lie algebras of type $E_{7}$. In the final section we classify completely all forms of $M(Y)$, $\mathcal{J}$ exceptional central simple, for finite, $p$-adic, or real fields.

In [5], symplectic algebras (an asymmetric version of FTS's) are studied, yielding different proofs of several results obtained in this paper.

Received by the editors December 22, 1971.

AMS (MOS) subject classifications (1970). Primary 17A30; Secondary $17 \mathrm{~B} 60$.

Key words and phrases. Freudenthal triple system, strictly regular element, Peirce decomposition, quadratic Jordan algebra, inner ideal.

(1) Research supported by NSF Grant GP-12247. 
We assume throughout that all algebras are finite dimensional and all fields are of characteristic $\neq 2,3$.

1. Construction of a class of FTS's. A Freudentbal Triple System is a vector space $\mathbb{M}$ with trilinear product $(x, y, z) \rightarrow x y z$ and skew bilinear form $(x, y) \rightarrow$ $\langle x, y\rangle$ such that

A1. $x y z$ is symmetric in all arguments;

A2. $q(x, y, z, w)=\langle x, y z w\rangle$ is a nonzero symmetric 4-linear form;

A3. $(x x x) x y=\langle y, x\rangle x x x+\langle y, x x x\rangle x$ for $x, y \in \mathbb{M}$.

A2 is equivalent to

$A 2^{\prime} .\left\langle x, y L_{z, z}\right\rangle=-\left\langle x L_{z, z}, y\right\rangle,\left\langle x, x L_{x x}\right\rangle \equiv 0$ where $L_{x, y}: z \rightarrow x y z$.

Linearizing A3 completely yields

$$
\begin{aligned}
(x u v) y z+ & (z u v) x y+(x u z) v y+(x v z) u y \\
= & \langle y, u\rangle x v z+\langle y, v\rangle x u z+\langle y, z\rangle x u v+\langle y, x\rangle u v z \\
& +\langle y, x v z\rangle u+\langle y, x u z\rangle v+\langle y, u v x\rangle z+\langle y, u v z\rangle x .
\end{aligned}
$$

The prototype FTS is constructed as follows: Let $\mathfrak{I}(N, 1)$ be the quadratic Jordan algebra of an admissible cubic form $N$ with basepoint $1, T(a, b)$ the associated trace form, $a \rightarrow a^{\#}$ the associated quadratic mapping and $a \times b=$ $(a+b)^{\#}-a^{\#}-b^{\#}$. By [10], assuming $T(a, b)$ nondegenerate, we have $a^{\sharp \#}=$ $N(a) a, N(1)=1, T(a \times b, c)=6 N(a, b, c)$. Define $M(\Im)=\left\{\left(a_{1}, a_{2}, a_{1}, a_{2}\right) \mid a_{i} \in F\right.$, $\left.a_{i} \in \mathfrak{S}\right\}$ and define on $\mathfrak{M}(\mathfrak{S})$ the forms

$$
\begin{aligned}
& \langle x, y\rangle=a_{1} \beta_{2}-a_{2} \beta_{1}+T\left(a_{1}, b_{2}\right)-T\left(a_{2}, b_{1}\right) . \\
& q(x)=12\left(4 a_{1} N\left(a_{1}\right)+4 a_{2} N\left(a_{2}\right)-4 T\left(a_{1}^{\#}, a_{2}^{\#}\right)+\left(T\left(a_{1}, a_{2}\right)-a_{1} a_{2}\right)^{2}\right)
\end{aligned}
$$

where $x=\left(\alpha_{1}, a_{2}, a_{1}, a_{2}\right), y=\left(\beta_{1}, \beta_{2}, b_{1}, b_{2}\right)$.

We let $q(x, y, z, w)$ denote the linearized form of $q(x)$ and define $x y z$ by

$$
q(w, x, y, z)=\langle w, x y z\rangle \text { for all } w^{\cdot} \epsilon \cdot M(\Im) .
$$

Since $($,$\rangle is clearly nondegenerate, the product is well defined. One easily$ sees that $A 1$ and $A 2$ are satisfied. That $A 3$ is valid follows directly from the arguments of Brown [1, Lemmas 9 and $10(\mathrm{~d})]$.

Since the proof of $\mathrm{A} 3$ depends only on the fact $a^{\# \#}=N(a) a$, we can construct a degenerate case $\mathfrak{M}(\mathfrak{S})$ as follows. Let $\Im(Q, c)$ be the Jordan algebra of the quadratic form $Q$ with basepoint $c[10], T(a, b)=Q\left(a, b^{*}\right)$, and define $a^{\#}=N(a)=0$ for all $a \in \mathfrak{I} . \mathbb{M}(\mathfrak{I})$ is once again a FTS with nondegenerate form $\langle$,$\rangle if Q$ is nondegenerate. The algebra obtained in this manner can also be realized in terms of a skew bilinear form on a vector space $M$ (see [9]).

For later use we establish 
1.1 Lemma. If $\mathfrak{J}_{0}=\mathfrak{S}(Q, c)$ is the Jordan algebra of a nondegenerate quadratic form, there is a quadratic Jordan algebra $\mathfrak{J}(N, 1)$ such that $\mathbb{M}\left(\mathfrak{J}_{0}\right)$ is is omorpbic to a subalgebra of $\mathbb{M}(\Im)$.

Proof. Let $\Re=\widetilde{S}_{0} \oplus \widetilde{J}_{0} t$ be a sum of copies of $\mathfrak{I}_{0}, \widetilde{\Im}_{0}=F u+\Re$. Define a quadratic form $Q_{0}$ on $\widetilde{\mathfrak{J}}_{0}$ by $Q_{0}(a u+a+b t)=a^{2}-Q\left(a^{*}, b\right), Q_{0}$ is a nondegenerate quadratic form and the Jordan algebra $\widetilde{S}=F e \oplus \widetilde{\mathfrak{S}}_{0}$ with cubic form $N$ as in [10, p. 506] and basepoint $e$ is the algebra of an admissible form. Moreover, it is easy to check that $\phi:(\alpha, \beta, a, b) \rightarrow(\alpha, \beta, a, b t), a, b \in \widetilde{J}_{0}$, is a linear transformation of $\mathfrak{M}\left(\mathfrak{S}_{0}\right)$ into $\mathfrak{M}(\mathfrak{S})$ which preserves both $\langle-,-\rangle$ and $q(-)$, hence is an isomorphism.

2. Ideals in Freudenthal triple systems. A subspace $\Re$ of a FTS $\mathfrak{M}$ is an inner ideal if $\Re \mathbb{M} \subseteq \Re$, an ideal if $\mathbb{R} \Re \subseteq \Re$. Clearly ideals are inner, but the converse can be shown to be false. $\mathbb{M}$ is simple if there are no proper ideals in $\mathbb{M}$.

Simple FTS's are characterized by

2.1 Theorem. $\mathbb{M}$ is simple iff $\langle-,-\rangle$ is nondegenerate.

Proof. If $x \in \Re=$ radical of $\langle-,-\rangle, 0=\left\langle x, y L_{z, w}\right\rangle=-\left\langle x L_{z, w}, y\right\rangle$ for all $y \in \Re$ by $A 2^{\prime}$, thus $\Re$ is an ideal. Conversely, assume $\Re \neq\{0\}$ is a proper ideal of $\mathfrak{M}$. For $y \in \mathbb{M}$ with $q(y) \neq 0$, (1) with $u=v=y=z$ yields $3 x L_{y y}^{2}=$ $2\langle y, x\rangle y y y+2\langle x, y y y\rangle y+q(y) x$. Writing $\mathbb{R}=F y \oplus F y y y \oplus(F y \oplus F y y y)^{\perp}$ and considering the action of $L_{y y}^{2}$ on summands yields

$$
L_{y y} \text { is nonsingular if } q(y) \neq 0 \text {. }
$$

An immediate consequence is that $\Re$ contains no element with $q(y) \neq 0$. Further, if $q(y) \neq 0$ we have $y y y \neq 0$. For $x \in \Re$ we consider $w=(y y y) y x=\langle x, y\rangle y y y+$ $\langle x, y y y\rangle_{y} \in \Re$ and note that $w L_{y y}=\langle x, y\rangle q(y) y+\langle x, y y y\rangle y y y \in \Re$ also. Since (1) implies

$$
q(y y y)=9 q(y)^{3}
$$

we have $y y y \notin \Re$. If either $\langle x, y\rangle$ or $\langle x, y y y\rangle$ is nonzero, we must thus have $w L_{y y}=k_{x} w, k_{x}=\langle x, y y y\rangle\langle x, y\rangle^{-1}$. Equating coefficients then yields $q(y)=k_{x}^{2}$ for any $x$ with $\langle x, y\rangle \neq 0$. Since $k_{x} y+y y y \in \Re$, previous remarks imply $0=q\left(k_{x} y+y y y\right)$, but direct computation of $q\left(k_{x} y+y y y\right)$. yields $4 q(y)^{3} \neq 0$, a contradiction. Thus for all $y \in \mathbb{M}$ with $q(y) \neq 0$ we have $\langle x, y\rangle=0$. A density argument then implies $x \in \Re$ so $\Re \subseteq \Re$.

2.2 Corollary (to proof). $\Re$ is the unique maximal ideal in $M$ and $M / \Re$ is simple.

2.3 Corollary. The systems $\mathbb{M}(\mathfrak{S})$ of $\$ 1$ are simple. 
While simple FTS's may contain inner ideals, we have the following restriction on their structure.

2.4 Theorem. Let $\mathbb{R}$ be a simple FTS, $\Re \subseteq M$ an inner ideal, $x, y \in \Re$. Then $\langle x, y\rangle=0=x x x$.

Proof. By (2), $q(z)=0$ for all $z \in \Re$ so by linearization $\langle w, z z z\rangle=0$ for all $w, z \in \Re$. If $\Re_{0}$ denotes the radical of $\langle-,-\rangle_{\mid \Re}$, for $x \in \Re_{0}, u \in \Re,\langle x x x, u\rangle=$ $\langle x x u, x\rangle=0$ so $x x x \in \Re=\{0\}$. If $x \notin \Re_{0}, w \in \Re$ with $\langle x, w\rangle \neq 0,(x x x) x w=$ $\langle w, x\rangle x x x$ by $\mathrm{A} 3$ and the above remark. Now for any $u \in \mathbb{R},\langle w, x\rangle\langle u, x x x\rangle=$ $\langle u,(x x x) x w\rangle=-\langle u x w, x x x\rangle=0$ so $x x x \in \Re=\{0\}$ and the triviality of the product is established.

Assume now there are $x, y \in \Re$ with $\langle x, y\rangle \neq 0$. Then $u L_{x, x} L_{y, x}=$ $\langle y, x\rangle u L_{x, x}+\left\langle y, u L_{x, x}\right\rangle x$ for all $u \in M$ by (1). Since the product in $\Re$ is trivial $\dot{u} L_{x, x} L_{y, x}=0$ and $\left\langle y, u L_{x, x}\right\rangle=0$. Thus $\langle y, x\rangle_{u L_{x, x}}=0$ so $L_{x, x} \equiv 0$.

From (1) with $z=x$ we have for all $u, v, y \in \mathbb{M}$,

$$
(x u v) x y=\langle y, x\rangle x u v+\langle y, x u v\rangle x
$$

so, for $u, z \in \mathbb{M}$ with $\langle z, x\rangle=\langle u, x\rangle=0$ we have $\langle y, x\rangle\langle z, x u u\rangle=\langle z,(x u u) x y\rangle=$ $\langle(x z y) x u, u\rangle=0$, the last equality following from expansion of $(x z y) x u$ via (4).

Again assuming $\langle x, y\rangle \neq 0$ we have $\left\langle x L_{u, u}, z\right\rangle=0$ for all $z, u \in(F x)^{\perp}$ and, since $\langle$,$\rangle is nondegenerate x L_{u, u}=a_{u} x, a_{u} \in F$. From (4) with $v=u, 0=$ $2 a_{u}\langle y, x\rangle x$ so $\alpha_{u}=0$. Now for any $v \in \mathbb{M},\left\langle v L_{u, u}, x\right\rangle=0$ so $\mathbb{M L}_{u, u} \subseteq(F x)^{\perp}$ for all $u \in$ $(F x)^{\perp}$ and $(F x)^{\perp}$ is an inner ideal. Moreover, for all $z, w \in M,\left\langle x L_{z, w}, x\right\rangle=\langle x x z, w\rangle=0$ since $L_{x, x}=0$ so $F x M \cap \cap \subseteq(F x)^{\perp}$. For $y \in M$ with $q(y) \neq 0$ this implies $\langle x, y\rangle \neq 0$, $\langle x, y y y\rangle \neq 0$ and $(y y y) y x=\langle x, y\rangle y y y+\langle x, y y y\rangle y \in(F x)^{\perp}$. Taking inner products with $x$ yields $0=2\langle x, y\rangle\langle x, y y y\rangle$, a contradiction. Thus we have for all $x, y \in \Re$, $\langle x, y\rangle=0$ as desired.

2.5 Corollary (to proof). Let $\Re$ be a simple FTS, $x \in M$ such that $L_{x, x}=0$. Then $x=0$.

We remark that for symplectic algebras, the analogue of Theorem 2.1 has been proved in [2].

Henceforth we restrict attention to simple FTS's.

3. Strictly regular elements in simple FTS's. $u \in M$ is strictly regular if $\Re L_{u, u} \subseteq F u$. Such elements play a role in the structure theory of FTS's analogous to that of idempotents in nonassociative algebras with identities. If $u$ is strictly regular, (2) implies $q(u)=0$, so $0=q(u) u=u L_{u, u}^{2}=\beta_{u}^{2} u$ where $u u x=\beta_{x} u$. Thus $\beta_{u}=0$. Now taking $x=u=z$ in (1) we obtain $\beta_{v} \beta_{y} u=2\langle y, u\rangle \beta_{v} u$ and since, by Corollary 2.5 there is $v \in \mathbb{M}$ with $\beta_{v} \neq 0$ we have 


$$
\begin{aligned}
y L_{u, u} & =2\langle y, u\rangle u, \\
q(u, u, x, y) & =2\langle y, u\rangle\langle x, u\rangle \text { for all } x, y \in \mathbb{M} .
\end{aligned}
$$

In determining explicit forms of strictly regular elements for Corollary 6.3 we shall need

3.1 Lemma. $u \in \mathbb{M}$ is strictly regular if and only if $u u u=0$ and $u \epsilon$ Range $L_{u, u}$.

Proof. Clearly strictly regular elements have the desired properties. Conversely, if $v \in \mathbb{M}$ with $v u u=u$, (1) implies for all $y \in \mathbb{M}, y L_{u, u}=2\langle y, u\rangle u$, so $u$ is strictly regular.

We have the useful

3.2 Corollary. If $\mathbb{M}_{1}$ is a simple subsystem of the simple system $M_{,} u \in M_{1}$ is strictly regular in $\mathbb{M}_{1}$ if and only if it is strictly regular in $\mathbb{M}$.

Proof. The lemma clearly implies if $u$ is regular in $\mathbb{R}_{1}$ it is regular in $\mathbb{M}$. Conversely, if it is regular in $\Re$, either $u \in M_{1} u u$ or $\Re_{1} u u=0$, the latter being impossible by Corollary 2.5.

We call $M$ reduced if $M$ contains a strictly regular element $u$. In such a system one can show by straightforward calculation, using (5) and (1) repeatedly, that for $w \in M$ with $\langle w, u\rangle \neq 0, v=3 u L_{w, w}+6\langle u, w\rangle w+\langle u, w\rangle^{-1}\langle u, w w w\rangle u$ is a strictly regular element in $\mathbb{M}$ satisfying $\langle u, v\rangle=3\left\langle u, u L_{w, w}\right\rangle+6\langle u, w\rangle^{2}=$ $12\langle u, w\rangle^{2} \neq 0$. A pair of strictly regular elements are supplementary if $\langle u, v\rangle=1$. After replacing $v$ in the above argument by a suitable scalar multiple we have proved

3.3 Theorem. Let $M$ be a simple FTS. Then $M$ is reduced if and only if $M$ contains a pair of supplementary, strictly regular elements.

3.4 Corollary. $M$ is reduced if and only if $M$ contains an element $x$ with $q(x)=12 \beta^{2}, \beta \in F^{*}$.

Proof. If $u, v$ are supplementary, strictly regular elements, $q(u+v)=12$. Conversely, if $q(x)=12 \beta^{2}$, direct computation using (1) shows $\left(\beta^{-1 / 12}\right)(x x x)+$ $1 / 2 x=u,\left(-\beta^{-1} / 12\right)(x x x)+1 / 2 x=v$ are strictly regular with $\langle u, v\rangle=-\beta$. After suitable scaling, we see that $\Re$ contains a pair of supplementary strictly regular elements.

3.5 Corollary. If $F$ is algebraically closed, every simple FTS over $F$ is reduced.

In studying the groups related to $M$ in $\$ 7$, we need 
3.6 Lemma. If $x \in M$ satisfies $q(x)=12 \beta^{2}, x$ is uniquely expressible as the sum of two (nonorthogonal) strictly regular elements.

Proof. The elements $u, v$ of the proof of Corollary 3.4 give one pair of such summands. If $x=u^{\prime}+v^{\prime}$ is another such expression, $\left\langle u^{\prime}, v^{\prime}\right\rangle=\alpha$, direct computation yields $u^{\prime}=1 / 2 x-\left(\alpha^{-1} / 12\right)(x x x), v^{\prime}=1 / 2 x+\left(\alpha^{-1} / 12\right)(x x x)$ and thus $\alpha= \pm \beta$, which yields the uniqueness.

In the case $F$ not algebraically closed we have

3.7 Corollary. Let $x \in \mathbb{M}, q(x) \neq 0, \mathbb{M}_{x}=F x+F(x x x)$. Then $\mathbb{M}_{x}$ contains either zero or two elements (projectively) with www $=0$. If such elements exist they are strictly regular and span the space $\mathbb{M}_{x}$.

Proof. Let $\Omega$ be an algebraic closure of $F$. In $\mathfrak{M}_{\Omega}$ we have $x=u+v, u, v$ supplementary strictly regular elements spanning $\left(\mathbb{M}_{x}\right)_{\Omega}$. Moreover, $q(\alpha u+\beta v) \neq 0$ if $\alpha \beta \neq 0$, hence $(\alpha u+\beta v)(\alpha u+\beta v)(\alpha u+\beta v) \neq 0$ unless $\alpha$ or $\beta=0$. Thus at most two such $w$ exist and they are strictly regular. Moreover, if $x+\alpha x x x \in \mathbb{M}_{x}$ is strictly regular, $\alpha= \pm \beta^{-1} / 6$ where $q(x)=12 \beta^{2}$ (in $\Omega$ ), hence $\beta \in F$ and $x-\operatorname{axx} \in \mathbb{M}_{x}$ as desired.

We note that if one considers the FTS $\mathbb{M}$ obtained from the system of Freudenthal [7] by symmetrizing the ternary product, the strictly regular elements turn out to be precisely the elements of the manifold related to the group $E_{7}$. Also, it is a consequence of Corollary 6.2 that the rank one elements of the symplectic algebra used in the study of an $E_{7}$ geometry [3] are precisely the strictly regular elements of the symmetrized algebra.

4. The Peirce decomposition of a simple, reduced FTS. If $u_{1}, u_{-1}$ are supplementary, strictly regular elements of $M,(1)$ and (5) imply

$$
x L_{u_{1}, u_{2}}^{2}=-3\left\langle x, u_{1}\right\rangle u_{-1}+3\left\langle x, u_{-1}\right\rangle u_{1}+x \text {. }
$$

Thus on $\left(F u_{1} \oplus F u_{-1}\right)^{\perp}$ we have $L_{u_{1}, u_{2}}^{2}=\mathrm{Id}$, so $\left(F u_{1} \oplus F u_{-1}\right)^{\perp}=\mathbb{M}_{1} \oplus \mathbb{M}_{-1}$, where $\mathbb{M}_{\epsilon}$ is the eigenspace for the eigenvector $\epsilon$ of $L_{u_{1}, u_{-1}}$. Moreover, since $\langle-,-\rangle$ is nondegenerate and its restriction to $F u_{1} \oplus F u_{-1}$ is nondegenerate, we have

$$
M=F u_{1} \oplus F u_{-1} \oplus \mathbb{M}_{1} \oplus \mathbb{M}_{-1}=\mathbb{M}_{2} \oplus \mathbb{M}_{-2} \oplus \mathbb{M}_{1} \oplus \mathbb{M}_{-1}
$$

since (5) implies $F u_{\epsilon}$ is the eigenspace for $L_{u_{1}, u_{-1}}$ with eigenvalue $-2 \epsilon$.

$A 2^{\prime}$ and the fact that $\langle-,-\rangle$ is skew imply that all Peirce spaces are totally isotropic. The nondegeneracy of $\left.\langle-,-\rangle\right|_{\mathbb{N}_{1} \oplus \mathbb{N}_{-1}}$ then implies $\operatorname{dim} \mathbb{M}_{1}=\operatorname{dim} \mathbb{M}_{-1}$.

For $\epsilon= \pm 1$ we have

4.1 Lemma. (i) $\mathbb{M}_{\epsilon} \mathbb{M}_{2 \epsilon} \mathbb{M}_{-2 \epsilon} \subseteq M_{\epsilon}$; 
(ii) $\mathbb{M}_{\epsilon} \mathbb{R}_{2 \epsilon} \mathbb{M}_{2 \epsilon}=\mathbb{M}_{-\epsilon} \mathbb{M}_{2 \epsilon} \mathbb{M}_{2 \epsilon}=\{0\}$;

(iii) $\mathbb{N}_{\epsilon} \mathbb{M}_{\epsilon} \mathbb{M}_{2 \epsilon}=\{0\}$;

(iv) $\mathbb{M}_{\epsilon} \mathbb{M}_{-2 \epsilon} \subseteq M_{-\epsilon}$;

(v) $\mathbb{M}_{\epsilon} \mathbb{M}_{-\epsilon} \mathbb{M}_{2 \epsilon} \subseteq \mathbb{M}_{2 \epsilon}$;

(vi) $\mathbb{M}_{\epsilon} \mathbb{M}_{\epsilon} \mathbb{M}_{\epsilon} \subseteq \mathbb{M}_{2 \epsilon}$;

(vii) $\mathbb{M}_{\epsilon} \mathbb{M}_{\epsilon} \mathbb{M}_{-\epsilon} \subseteq \mathbb{M}_{\epsilon}$.

Proof. (i) is direct from the definition while (ii) follows from the definitions and the orthogonality relations among the Peirce spaces. For (iii) we expand $x_{\epsilon} y_{\epsilon}^{u}-\epsilon$ using $\epsilon\left(x_{\epsilon}^{u} 1^{u}-1\right)=x_{\epsilon}$ and (1) to obtain $4 \epsilon x_{\epsilon}^{u}-\epsilon^{x} \epsilon=0$. Since (1) also yields $\left(x \epsilon^{x} \epsilon_{\epsilon}\right) u_{\epsilon} u_{-\epsilon}=-\epsilon\left(x_{\epsilon}^{x} \epsilon_{\epsilon}\right)$ we have (iv). (v) now follows directly from A2', the preceding results and the orthogonality relations. (vi) again follows from an expansion of $x_{\epsilon} x_{\epsilon} x_{\epsilon}$ using $\epsilon x_{\epsilon}=x_{\epsilon} u_{1} u_{-1}$, (1), the orthogonality relations and previous results. ( $v$ ii) is then a direct consequence of the orthogonality relations. A2',

From (v) we obtain immediately, by taking inner products with $u_{\epsilon}$ and using

$$
x_{\epsilon} y_{-\epsilon} u_{-\epsilon}=-\left\langle x_{\epsilon}, y_{-\epsilon}\right\rangle u_{-\epsilon} .
$$

For the coordinatization theorem of $\$ 5$ we need a means of identifying the spaces $\mathbb{M}_{1}$ and $\mathbb{M}_{-1}$ in a manner consistent with the forms $q(-),\langle-,-\rangle$. We define $t: \mathbb{M}_{1} \rightarrow \mathbb{M}_{-1}$ as follows: if, for all $y \in \mathbb{M}_{1},\left\langle u_{1}, y y y\right\rangle=0$, let $a_{1}, \ldots, a_{n}$ be a basis for $\mathbb{M}_{1}, a_{1}^{\prime}, \ldots, a_{n}^{\prime}$ a dual basis for $\mathbb{M}_{-1}$ relative to $\langle-,-\rangle$ and define $t$ by $a_{i} t=2 a_{i}^{\prime}$; if there is $y \in \mathbb{M}_{1}$ with $\left\langle u_{1}, y y y\right\rangle=\lambda \neq 0$, define $t$ by $a t=-1 / 2 a y u_{1}+3 / 4 \lambda^{-1}\left\langle u_{1}, y y a\right\rangle y y u_{1}$. We then have

4.2 Lemma. For all $a, b \in \mathbb{M}_{1}$,

(i) $\langle a, b t\rangle=-\langle a t, b\rangle$;

(ii) $\left\langle u_{-1}\right.$, atatat $\rangle=(1 / 12) \lambda\left\langle u_{1}, a a a\right\rangle$;

(iii) $t$ is nonsingular.

Proof. (i) is immediate from $A 2^{\prime}$ in the latter case and from the definition in the former.

In the case $y$ exists with $\left\langle u_{1}, y y y\right\rangle=\lambda \neq 0$ one can use $A 2^{\prime}$ to obtain $\left\langle\left(u_{1} y a\right)\left(u_{1} y a\right)\left(u_{1} y a\right), u_{-1}\right\rangle=\left\langle u_{1},\left(\left(u_{1} y a\right)\left(u_{1} y a\right) u_{-1}\right) y a\right\rangle$ and then expand $\left(\left(u_{1} y a\right)\left(u_{1} y a\right) u_{-1}\right) y a$ using (1), A2', (7) and the orthogonality relations to obtain

$$
\left(u_{1} y a\right)\left(u_{1} y a\right)\left(u_{1} y a\right)=\left(-6\left\langle y a a, u_{1}\right\rangle\left\langle y y a, u_{1}\right\rangle+(2 / 3)\left\langle y y y, u_{1}\right\rangle\left\langle a a a, u_{1}\right\rangle\right) u_{-1}
$$

for all $a \in \mathbb{M}_{1}$. Linearizing yields

$$
\begin{aligned}
\left(u_{1} y a\right)\left(u_{1} y a\right)\left(u_{1} y b\right) & \\
=\left(-4\left\langle y a b, u_{1}\right\rangle\left\langle y y a, u_{1}\right\rangle\right. & -2\left\langle y a a, u_{1}\right\rangle\left\langle y y b, u_{1}\right\rangle \\
& \left.+(2 / 3)\left\langle y y y, u_{1}\right\rangle\left\langle a a b, u_{1}\right\rangle\right) u_{-1} .
\end{aligned}
$$


(ii) then follows by direct computation with repeated use of (8). In this case also we derive from (1), Lemma 4.1 , and the orthogonality relations $3(y y x) u_{1} y=$ $2\left\langle u_{1}, y y y\right\rangle x$ for all $x \in \mathbb{M}_{-1}$. Thus $L_{u_{1}, y}$ is surjective, hence bijective from $\mathbb{M}_{1}$ to $\mathbb{M}_{-1}$. Since $y t \neq 0$ for $y$ as above, we thus see that $t$ is also bijective.

As a consequence of (ii) and (iii) in the case $\left\langle y y y, u_{1}\right\rangle \equiv 0$, we have $\left\langle u_{-1}, b b b\right\rangle=0$ for all $b \in M_{-1}$ if $\left\langle y y y, u_{1}\right\rangle \equiv 0$. Thus (ii) and (iii) follow trivially in this case.

4.3 Corollary. For $t$ as above, $x, z \in \mathbb{M}_{1},\left(x t z t u_{-1}\right) t=(-\lambda / 12)\left(x z u_{1}\right)$.

Proof. In case $\left\langle u_{1}, y y y\right\rangle \equiv 0$ on $\mathbb{M}_{1}$, the nondegeneracy of $\langle-,-\rangle$ and the fact that $\Re_{1}, M_{-1}$ are dual relative to the form imply both sides are zero. In the remaining case, it suffices to compute inner products with all $w \in M_{1}$, but by the lemma

$$
\begin{aligned}
\left\langle w,\left(x t z t u_{-1}\right) t\right\rangle= & -\left\langle w t, x t z t u_{-1}\right\rangle=-\left\langle u_{-1}, x t z t w t\right\rangle \\
& =(-\lambda / 12)\left\langle u_{1}, x z w\right\rangle=(-\lambda / 12)\left\langle w, x z u_{1}\right\rangle \text { as desired. }
\end{aligned}
$$

We note finally that (1), Lemma 4.1 and the orthogonality relations yield

$$
\left(x x u_{1}\right) u_{-1}\left(x x u_{1}\right)=-(16 / 3)\left\langle u_{1}, x x x\right\rangle x \text { for all } x \in \mathbb{M}_{1}
$$

and

$$
x x y=2\langle y, x\rangle x-1 / 2\left(x x u_{1}\right) u_{-1} y \quad \text { for } x \in \mathbb{M}_{1}, y \in M_{-1} .
$$

5. Coordinatization of simple reduced FTS's. Using the results of $\$ 4$ we prove, in a manner analogous to that in [1],

5.1 Theorem. Let $M$ be a reduced, simple FTS over $F_{0}$. Then $M \cong M(\Im)$ (see $\$ 1$ ) where $\mathfrak{S}$ is

(i) the quadratic Jordan algebra of an admissible cubic form with basepoint,

or

(ii) the quadratic Jordan algebra of a nondegenerate quadratic form.

For proof, we show that the Peirce space $\mathbb{M}_{1}$ can be endowed with the structure of a Jordan algebra of one of the above types in such a way that the map $\phi: a u_{1}+\beta u_{-1}+a+b t \rightarrow(\alpha, \beta, a, b), t$ as in $\$ 4$, is an isomorphism of triple systems. In particular, it will suffice to show $\phi$ is an isometry relative to both the skew bilinear forms and the quartic forms (see Lemma 6.6). As in $\$ 4$ we begin with a pair $\left(u_{1}, u_{-1}\right)$ of supplementary, strictly regular elements and distinguish cases: (i) $\left\langle u_{1}, x x x\right\rangle \equiv 0$ on $\mathbb{M}_{1}$, (ii) $\left\langle u_{1}, x x x\right\rangle \equiv 0$ on $\mathbb{M}_{1}$. In case (ii) we pick $y \in M_{1}$ with $\left\langle u_{1}, y y y\right\rangle=12 \lambda \neq 0$ and in case (i) we set $\lambda=1$. Using $t$ as in $\$ 4$ we define $T(a, b)=\lambda^{-1}\langle a, b t\rangle$ and $N(a)=\left(\lambda^{-1} / 12\right)\left\langle u_{1}, a a a\right\rangle$ on $M_{1}$. 
The nonsingularity of $t$ and nondegeneracy of $\langle-,-\rangle$ imply $T(-,-)$ is nondegenerate and Lemma 4.2(i) implies it is symmetric. We can thus define a commutative product on $\mathbb{M}_{1}$ by $T(a \times b, c)=6 N(a, b, c)$. Lemma 4.2 and the nondegeneracy of $T(a, b)$ yields $a \times b=-\left(\lambda^{-1 / 2}\right)\left(a t b t u_{-1}\right)$. Together with Corollary 4.3 this implies $(a \times a) \times(a \times a)=8 N(a) a$.

Defining $a^{\#}=1 / 2 a \times a$ we thus have $a^{\# \#}=N(a) a$. Moreover, in case (ii) a straightforward calculation using the form of $t$ yields $T(a, b)=-\left.\partial_{a} \partial_{b} \log N\right|_{y}$. Since by definition $N(y)=1, \Re_{1}(N, y)$ is a quadratic Jordan algebra of the cubic form $N$, basepoint $y[10]$.

In the first case we consider $M$ as Jordan algebra of a quadratic form $Q$ with basepoint $a_{1}$ where $Q$ is defined as follows: let $*$ denote the mapping $a_{1} a_{1}+$ $\Sigma_{i>1} a_{i} a_{i} \rightarrow a_{1} a_{1}-\Sigma_{i>1} \alpha_{i} a_{i}$ and set $Q(a)=1 / 2 T\left(a, a^{*}\right)$. The Jordan trace form is then just $T(a, b)$. In this case we clearly have $N(a)=a^{\#}=0$ for all $a \in M_{1}$.

An inspection of the known orthogonality relations, (10), Lemma 4.1 and the definitions yields, for $x_{i}=a_{i} u_{1}+\beta_{i} u_{-1}+a_{i}+b_{i} t,\left\langle x_{1}, x_{2}\right\rangle=\alpha_{1} \beta_{2}-\beta_{1} \alpha_{2}+$ $\lambda\left(T\left(a_{1}, b_{2}\right)-T\left(b_{1}, a_{2}\right)\right)$,

$$
q\left(x_{1}\right)=12\left(-4 \lambda^{2} T\left(a_{1}^{\#}, b_{1}^{\#}\right)+4 \lambda \alpha_{1} N\left(a_{1}\right)+4 \lambda^{2} \beta_{1} N\left(b_{1}\right)+\left(\lambda T\left(a_{1} b_{1}\right)-a_{1} \beta_{1}\right)^{2}\right) .
$$

Replacing the pair $\left(u_{1}, u_{-1}\right)$ by $\left(\lambda^{-1} u_{1}, \lambda u_{-1}\right)$ (which again are supplementary, strictly regular elements) we may assume $\lambda=1$ so a comparison with the forms of $\$ 1$ yields the result.

6. Uniqueness of the coordinate algebra. An element $x \in \mathbb{M}$ is rank one if $L_{x, x}$ has one dimensional image. Among the rank one elements are the strictly regular elements. In this section we determine the form of all rank one elements in $\mathfrak{M}(\mathfrak{S}), \mathfrak{S}=\mathfrak{S}(N, 1)$, and use the result to study the coordinate algebra in an arbitrary reduced simple FTS.

The results of Springer [11], [12] show that if $\operatorname{dim} \mathfrak{S} \geq 3, \mathfrak{J}=\mathfrak{J}(N, 1)$ is either central simple of degree 3 or of form $\mathfrak{S}=F e+\mathfrak{I}_{0}, \mathfrak{I}_{0}$ the Jordan algebra of a quadratic form $Q_{0}$ [10]. We need the following facts about these algebras.

(11) If $a^{\#}=b^{\#}=0$, there is $c \in \mathfrak{J}$ with $c^{\#}=0$ such that $(a \times c) \times b \neq 0$.

(12) If $0 \neq a \in \mathfrak{J}, \operatorname{dim} a \times \mathfrak{I}>1$.

For algebras $\mathrm{Fe}+\mathfrak{S}_{0}$, (11) and (12) are consequences of elementary computations using the explicit form of $a^{\#}$ (given e.g. in [10, p. 506]). For central simple algebras, (12) follows from 1.1(a) of [4]. For (11), since $a \neq 0, a^{\#}=0$, $\Im$ must be reduced, hence spanned by elements $c$ with $c^{\#}=0$. It thus suffices to show $(a \times \Im) \times b \neq 0$. For this we can clearly use a field extension argument to assume $F$ algebraically closed, hence $\widetilde{S}$ split. The transitivity of the norm preserving group on $\left\{c \in \mathfrak{S} \mid c^{\#}=0\right\}$, together with the easily established fact that $\left(e_{1} \times \mathfrak{J}\right) \times b \neq 0$ for $0 \neq b \in \mathfrak{S}, e_{1}$ denoting the first diagonal idempotent in a coordinatization of $\mathfrak{S}$, gives the result. 
We can now establish

6.1 Lemma. Let $\mathfrak{S}=\mathfrak{J}(N, 1)$ be as above, $\operatorname{dim} \mathfrak{I} \geq 3$. $x \in \mathbb{M}(\mathfrak{S})$ is rank one if and only if $x=\left(a_{1}, a_{-1}, a_{1}, a_{-1}\right), a_{\epsilon} \in F, a_{\epsilon} \in \mathcal{J}$ where either

(i) for some $\epsilon, a_{\epsilon} \neq 0$ and $a_{\epsilon}=\alpha_{\epsilon}^{-1} \alpha_{-\epsilon}^{\#}, a_{-\epsilon}=\alpha_{\epsilon}^{-2} N\left(a_{-\epsilon}\right)$ or

(ii) $a_{1}=a_{-1}=0, a_{\epsilon}^{\#}=0$ and if $a_{-\epsilon} \neq 0, a_{\epsilon}=\left(a_{-\epsilon} \times z_{-\epsilon}\right)$, some $z_{-\epsilon} \epsilon \Im$.

Proof. The results of $\S_{5}$ imply $u_{1}=(1,0,0,0), u_{-1}=(0,1,0,0)$ are supplementary, strictly regular elements in $\mathscr{M}(\Im)$ and the related Peirce spaces are $\mathbb{M}_{2 \epsilon}=F u_{-\epsilon}, \mathbb{M}_{1}=(0,0, \Im, 0), M_{-1}=(0,0,0, \Im)$. Using the results of $\S_{4}$ to evaluate products we obtain

$$
u_{-\epsilon} x x=\left(\beta_{1}, \beta_{-1}, b_{1}, b_{-1}\right)
$$

where

$$
\begin{array}{ll}
\beta_{-\epsilon}=4 \epsilon a_{\epsilon} a_{-\epsilon}-2 \epsilon T\left(a_{1}, a_{-1}\right), & \beta_{\epsilon}=-2 \epsilon \alpha_{\epsilon}^{2}, \\
b_{-\epsilon}=-2 \epsilon a_{\epsilon} a_{-\epsilon}, & b_{\epsilon}=2 \epsilon\left(a_{\epsilon} a_{\epsilon}-2 a_{-\epsilon}^{\#}\right),
\end{array}
$$

$$
c_{\epsilon} x x=\left(\beta_{1}, \beta_{-1}, b_{1}, b_{-1}\right)
$$

where

$$
\begin{aligned}
\beta_{-\epsilon} & =-2 \epsilon a_{-\epsilon} T\left(a_{-\epsilon}, c_{\epsilon}\right)+12 \epsilon N\left(c_{\epsilon}, a_{\epsilon}, a_{\epsilon}\right), \quad \beta_{\epsilon}=2 \epsilon a_{\epsilon} T\left(c_{\epsilon}, a_{-\epsilon}\right), \\
b_{\epsilon} & =2 \epsilon\left(a_{\epsilon} a_{-\epsilon} c_{\epsilon}-T\left(a_{-\epsilon}, c_{\epsilon}\right) a_{\epsilon}-T\left(a_{-\epsilon}, a_{\epsilon}\right) c_{\epsilon}+2\left(a_{\epsilon} \times c_{\epsilon}\right) \times a_{-\epsilon}\right), \\
b_{-\epsilon} & =2 \epsilon\left(2 a_{\epsilon}\left(a_{\epsilon} \times c_{\epsilon}\right)+T\left(c_{\epsilon}, a_{-\epsilon}\right) a_{-\epsilon}-2\left(a_{-\epsilon}^{\#} \times c_{\epsilon}\right)\right), \quad c_{\epsilon} \epsilon \mathbb{M}_{\epsilon} .
\end{aligned}
$$

Assume now $L_{x, x}$ has 1 -dimensional image.

Case 1. $a_{1} \alpha_{-1} \neq 0$. Then $u_{-\epsilon} x x=v \neq 0$, hence $c_{\epsilon} x x=\rho v, \rho \in F$. Equating the components from $\mathbb{N}_{-\epsilon}$ then yields $-\left(\rho a_{\epsilon}+T\left(c_{\epsilon}, a_{-\epsilon}\right)\right) a_{-\epsilon}=$ $c_{\epsilon} \times\left(2 a_{\epsilon} a_{\epsilon}-2 a_{-\epsilon}^{\#}\right)$. (12) then implies $a_{\epsilon} a_{\epsilon}-a_{-\epsilon}^{\#}=0$, one of the desired conditions. Moreover, we have $a_{1}=0$ if and only if $a_{-1}=0$ and since $x=$ $a_{1} u_{1}+a_{-1} u_{-1}$ clearly does not satisfy the condition on $L_{x, x}, a_{\epsilon} \neq 0, \epsilon= \pm 1$. The above equation also yields $\rho=-\alpha_{\epsilon}^{-1} T\left(c_{\epsilon}, a_{-\epsilon}\right)$ so, equating coefficients of $u_{-\epsilon}$ in (13) and (14) we have, for all $c_{\epsilon}, c \in \mathfrak{S}, 2 \epsilon \alpha_{\epsilon}^{-1} T\left(c_{\epsilon}, a_{-\epsilon}\right) T\left(a_{\epsilon}, a_{-\epsilon}\right)-$ $4 \epsilon \alpha_{-\epsilon} T\left(c_{\epsilon}, a_{-\epsilon}\right)=-2 \epsilon \alpha_{-\epsilon} T\left(a_{-\epsilon}, c_{\epsilon}\right)+4 \epsilon T\left(c_{\epsilon}, a_{\epsilon}^{\#}\right)$. The nondegeneracy of $T(-,-)$ and $a_{\epsilon}^{\#}=a_{-\epsilon} a_{-\epsilon}$ imply $3 \alpha_{-\epsilon} a_{-\epsilon}=\alpha_{\epsilon}^{-1} T\left(a_{\epsilon}, a_{-\epsilon}\right) a_{-\epsilon}=\alpha_{\epsilon}^{-1} \alpha_{-\epsilon}^{-1} T\left(a_{\epsilon}, a_{\epsilon}^{\#}\right) a_{-\epsilon}=$ $3 a_{-\epsilon}^{-1} \alpha_{\epsilon}^{-1} N\left(a_{\epsilon}\right) a_{-\epsilon}$ which completes the proof that $x$ is of type (i).

Case 2. $\alpha_{\epsilon} \neq 0, \alpha_{-\epsilon}=0$. Analyzing the Peirce components of $u_{ \pm \epsilon} x x$ gives immediately $u_{\epsilon} x x=0$, hence $T\left(a_{\epsilon}, a_{-\epsilon}\right)=0, a_{\epsilon}^{\#}=0$.

The argument from Case 1 also yields $\alpha_{\epsilon} a_{\epsilon}=a_{-\epsilon}^{\#}$. Since in this case $N\left(a_{-\epsilon}\right)=(1 / 3) \alpha_{\epsilon} T\left(a_{-\epsilon}, a_{\epsilon}\right)=0, x$ is again of type (i). 
Case 3. $a_{\epsilon}=0, \epsilon= \pm 1$. Clearly rank $x=1$ implies either $u_{\epsilon} x x=0$ or $u_{-\epsilon} x x=0$ so $T\left(a_{1}, a_{-1}\right)=0$. If $a_{-1}^{\#} \neq 0$, Im $L_{x, x}=F\left(0,0, a_{-1}^{\#}, 0\right)$, but then analysis of $c_{ \pm \epsilon} x x$ yields $N\left(c_{-1}, a_{-1}, a_{-1}\right)=0$ for all $c \in \Im$, hence $a_{-1}^{\#}=0$, a contradiction. Arguing similarly we obtain $a_{1}^{\#}=0$. Equating Peirce coordinates of $c_{ \pm \epsilon} x x$ thus yields $\rho T\left(c_{\epsilon}, a_{-\epsilon}\right) a_{-\epsilon}=T\left(a_{\epsilon}, c_{-\epsilon}\right) a_{-\epsilon}-2\left(a_{-\epsilon} \times c_{-\epsilon}\right) \times a_{\epsilon}$ (11) then implies that for suitably selected $c$ with $c^{\sharp}=0, a_{-\epsilon}=a_{\epsilon} \times\left(a_{-\epsilon} \times c_{-\epsilon}\right)=$ $a_{\epsilon} \times z_{\epsilon}$ as desired. If $a_{-\epsilon}=0$, the conditions are satisfied by $z_{\epsilon}=0$.

Conversely, if $x$ has the form (i) or (ii), a straightforward calculation using (13), (14) and well-known identities (e.g. [4, 1.1-1.6]) relating $T(-,-), N(-)$ and $x$ yields in each case $y x x=2\langle y, x\rangle x$ for all $y \in \mathbb{M}(\mathfrak{S})$ so $x$ is rank 1 .

6.2 Corollary. If $\mathfrak{S}=\mathfrak{S}(N, 1)$, $\operatorname{dim} \mathfrak{\Im} \geq 3, x \in \mathbb{M}$ is strictly regular if and only if it is rank one.

Proof. We have already observed that strictly regular elements are rank one and the final observation of the proof of the lemma, together with the lemma, implies every rank one $x$ satisfies $y x x=2\langle y, x\rangle$, hence $x$ is strictly regular.

Corollary 6.2 is false if $\mathfrak{J}$ is the Jordan algebra of a nondegenerate quadratic form, as $x=a_{1}+b_{-1}, T(a, b)=0$, is easily seen from (13) and (14) to be rank one but not strictly regular.

6.3 Corollary. If $\mathfrak{M}$ is reduced, simple, $\mathfrak{M}$ is spanned by strictly regular elements.

Proof. It suffices to assume $\mathbb{R}=\mathfrak{M}(\mathfrak{S})$. If $\mathfrak{J}=\mathfrak{J}(N, 1)$, a density argument shows $\mathfrak{J}$ is spanned by elements $a$ with $N(a) \neq 0$. $\mathscr{M}(\mathcal{J})$ is then clearly spanned by $\left\{u_{1}, u_{-1}\right\} \cup\left\{a_{\lambda, \epsilon} \mid \lambda \in F\right\}$ where $a_{\lambda, \epsilon}$ is as in Lemma 6.1(i) with $a_{\epsilon}=\lambda$,

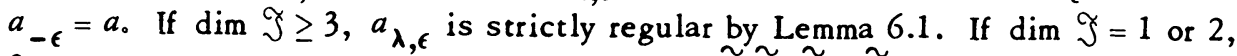
$\widetilde{J}$ is isomorphic to a subalgebra of an algebra $\widetilde{\mathfrak{J}}(\tilde{N}, \tilde{c}), \widetilde{\mathfrak{J}}$ of dimension 3 . Corollary 3.2 then implies $a_{\lambda, \epsilon}$ is strictly regular in $\mathscr{M}(\mathfrak{S})$. If $\mathfrak{S}$ is Jordan algebra of a quadratic form, $a_{1}, \ldots, a_{n}$ a basis for $\mathfrak{J}, \mathfrak{M}(\mathfrak{S})$ is spanned by $\left\{u_{1}, u_{-1},\left(0,0, a_{i}, 0\right),\left(0,0,0, a_{i}\right)\right\}$ where the latter elements are seen to be strictly regular in $M(\Im)$ by imbedding $M(\Im)$ in $M(\widetilde{\Im}), \widetilde{\widetilde{J}}=\widetilde{\Im}(\widetilde{N}, \tilde{1})$ by Lemma 1.1 and applying Lemma 6.1 and Corollary 3.2.

6.4 Corollary. If $\mathfrak{M}$ is simple, $x, y \in \mathbb{M}$ strictly regular, there are strictly regular elements $x=x_{0}, x_{1}, \cdots, x_{n}=y$ such that $\left\langle x_{i}, x_{i+1}\right\rangle \neq 0$.

Proof. By recoordinatizing we may assume $x=u_{1}, y=\left(a_{1}, a_{-1}, a_{1}, a_{-1}\right)$. If $a_{-1} \neq 0, n=1$ suffices. If $a_{1} \neq 0, n=2, x_{1}=u_{-1}$ suffices. Thus we assume $a_{1}=a_{-1}=0$. If $a_{-1} \neq 0$, pick $b \in \mathfrak{S}$ such that $T\left(b, a_{-1}\right) \neq 0$. For suitable choice of $\lambda, b_{\lambda,-1}=x_{1}, n=2$ suffices. Finally, if $a_{-1}=0, a_{1} \neq 0$ there is $c \in \mathfrak{S}$ such that $T\left(c, a_{1}\right)=0$. Taking $x_{n-1}=c_{1,1}$ yields $\left(x_{n-1}, x_{n}\right) \neq 0$ and 
by the above arguments $x_{i}, i<n-1$, exist satisfying the conditions.

Suppose now $M$ is reduced, simple, $u_{1}, u_{-1}$ a pair of supplementary strictly regular elements. The Jordan algebra structure coordinatizing $M(\$ 5)$ is largely determined by the trilinear form $N_{u_{1}, u_{-1}}(a, b, c)=\left\langle u_{1}, a b c\right\rangle$ on the Peirce space $\mathbb{M}_{1}$. As a step in proving uniqueness of the coordinate algebra we have

6.5 Lemma. Let $\left(u_{1}, u_{-1}\right),\left(v_{1}, v_{-1}\right)$ be supplementary pairs of strictly regular elements in $\mathfrak{M}_{\text {。 }}$ Then $N_{u_{1}, u_{-1}}$ is equivalent to a scalar multiple of $N_{v_{1}, v-1}$.

Proof. We consider first the case $u_{1}=v_{1}$. For this, we note that the results of $\S_{4}$ imply that $\left(F u_{1}\right)^{\perp}=F u_{1} \oplus \mathbb{M}_{1} \oplus \mathbb{M}_{-1}$, $\mathbb{M}_{\epsilon}$ in the Peirce decomposition relative to $\left(u_{1}, u_{-1}\right)$. The cubic form $\widetilde{N}(x)=\left\langle u_{1}, x x x\right\rangle$ on $\left(F u_{1}\right)^{\perp}$ has radical $\left(=\left\{y \mid \widetilde{N}(y, x, x)=0 \forall x \in\left(F u_{1}\right)^{\perp}\right\}\right) F u_{1}+M_{-1}$ by Lemma 4.1. The form $N_{u_{1}, u_{-1}}(a, b, c)$ on $\mathfrak{M}_{1}$ is thus clearly equivalent to the form induced on $\left(F u_{1}\right)^{\perp / \operatorname{Rad}} \widetilde{N}$ by $\widetilde{N}$. Since this latter form is independent of the strictly regular element $u_{-1}$ it follows that $N_{u_{1}, u_{-1}}$ is equivalent to $N_{u_{1}, v_{-1}}$. We denote the form thus by $N_{u_{1}}$. By definition of $N_{u_{1}}$ it is clear that $N_{\alpha u_{1}}=\alpha N_{u_{1}}$ for a $\in F^{*}$. Also, if $\left(u_{1}, u_{-1}\right)$ are a supplementary pair of strictly regular elements, Lemma 4.2(ii) implies $N_{u_{-1}}$ is equivalent to a multiple of $N_{u_{i}}$. Now if $u_{1} \neq v_{1}$, Corollary 6.4 implies there exist strictly regular $u_{1}=x_{1}, x_{2}, \cdots, x_{n}=v_{1}$ such that $\left\langle x_{i}, x_{i+1}\right\rangle \neq 0$. The above remarks then imply there exist $\alpha_{1}, \ldots, \alpha_{n}$ in $F^{*}$ such that $N_{x_{i}}$ is equivalent to $a_{i+1} N_{x_{i+1}}$, which gives the desired result.

We need also

6.6 Lemma. Let $\mathbb{M}_{i}, i=1,2$, be simple FTS's。 $\phi: \mathbb{M}_{1} \rightarrow \mathbb{M}_{2}$ is an isomorphism if and only if $q_{2}(x \phi)=q_{1}(x)$ and $\langle x \phi, y \phi\rangle_{2}=\langle x, y\rangle_{1}$ for all $x, y \in M_{1}$.

Proof. Since $\mathbb{M}_{i}$ are simple, $\langle-,-\rangle_{i}$ are nondegenerate by Theorem 2.1 , hence $A 2$ implies that the product is completely determined by the forms and one direction is clear. If $\phi$ is an isomorphism, applying $\phi$ to $A 3$, considering A3 for $x=x \phi, y=y \phi$ and comparing when $x=y$ yields $q_{2}(x \phi)=q_{1}(x)$. If $x \neq y$, we obtain, upon subtracting the two resulting equations, $\left((y, x\rangle_{1}-\langle y \phi, x \phi\rangle_{2}\right) x \phi x \phi x \phi$ $=0$ for all $x, y$. In particular, if $y$ is fixed and $x$ satisfies $x \phi x \phi x \phi \neq 0$, $\langle y, x\rangle=\langle y \phi, x \phi\rangle$. A density argument then implies this result is valid for all $x, y$ 。

We let Aut $M$ denote the group of automorphisms of the simple system $\mathbb{M}$,

$$
\begin{aligned}
& G_{0}(\mathbb{M})=\{\phi \in \operatorname{Hom}(\mathbb{M}, \mathbb{M}) \mid q(x \phi)=q(x) \forall x \in \mathbb{M}\} \text { and } \\
& Q_{0}(\mathbb{M})=\{\phi \in \operatorname{Hom}(\mathbb{M}, \mathfrak{M}) \mid\langle x \phi, y \phi\rangle=\langle x, y\rangle \forall x, y \in \mathbb{M}\} .
\end{aligned}
$$

Then we have 
6.7 Corollary. Aut $\mathbb{M}=G_{0}(\mathfrak{M}) \cap Q_{0}(\mathfrak{M})$.

We can now prove

6.8 Theorem. $M(\Im) \cong M(\Omega)$ only if there is $\eta: \Im \rightarrow \Re$ a linear isomorphism such that $N_{\Omega}(a \eta)=\rho N_{\mathcal{S}}(a)$ for all $a \in \mathfrak{S}$, some $\rho \in F^{*}$.

Proof. Let $u_{1}(\Im)=(1,0,0,0), u_{-1}(\Im)=(0,1,0,0)$. Then $u_{\epsilon}(\Im)$ are supplementary strictly regular elements in $\mathbb{M}(\mathfrak{S})$. If $\phi: M(\Im) \rightarrow \mathfrak{M}(\Re)$ is an isomorphism, $\phi\left(u_{\epsilon}(\Im)\right)=v_{\epsilon}$ are supplementary, strictly regular in $\mathbb{M}(\Omega)$ by Lemma 3.1. It is easily seen, for example from (14), that $N_{u_{1}(\Im)}=12 N_{\Im}, N_{u_{1}(\Omega)}=$ $12 N_{\Re}, N_{u}$ as in Lemma 6.5. Since $\phi$ must preserve Peirce spaces, it must map $\mathfrak{R}(\Im)_{1}$ onto the Peirce 1 -space for the pair $\left(v_{1}, v_{-1}\right)$. Lemma 6.6, together with the definition of $N_{u}$ thus implies $N_{u_{1}(\Im)}$ is equivalent to $N_{v_{1}(\boldsymbol{\beta})}$. Lemma 6.5 then yields the desired result.

The converse of this theorem is proved in $\$ 7$.

6.9 Corollary. If $\mathfrak{S}$ is Jordan algebra of an admissible cubic form $N$ with basepoint $c$ and $\mathfrak{M}(\Im) \cong \mathfrak{M}(\Omega), \mathfrak{S}$ and $\Omega$ are isotopic.

Proof. By the theorem there exists $\eta: \Im \rightarrow \Omega$ such that $N_{\Omega}(a \eta)=\rho N_{\mathcal{S}}(a)$ for all $a \in \Im$. In particular, this implies $N_{\Omega} \not \equiv 0$, hence $\Omega$ is algebra of a nondegenerate cubic form. The results of [10] (Theorem 2 and final remarks of $\$ 4)$ imply that $\eta$ is an isomorphism from $\mathfrak{J}$ to $\AA^{\left((c \eta)^{-1}\right)}$.

7. Groups related to reduced simple FTS's. In this section we study the action of the group $G(\mathbb{M})=\left\{\eta \in \operatorname{Hom}(\mathbb{M}, \mathfrak{M}) \mid q(x \eta)=\rho q(x) \forall x \in \mathbb{M}\right.$, some $\left.\rho \in F^{*}\right\}$ on the triple system $\mathfrak{M}$. We call $\rho$ the ratio of $\eta$. In the case $\mathbb{M}=\mathbb{M}(\mathfrak{S}), \mathfrak{S}$ exceptional simple, this is an algebraic group of type $E_{7}$ [1]. In an auxiliary manner the group $Q(\mathbb{M})=\{\eta \in \operatorname{Hom}(\mathbb{M}, \mathfrak{M}) \mid\langle x \eta, y \eta\rangle=\delta\langle x, y\rangle$ for all $x, y \in \mathbb{M}$, some $\left.\delta \in F^{*}\right\}$ also arises.

We note first, as a consequence of the results of $\$ 6$

7.1 Lemma. If $x \in \mathbb{M}$ is rank one, $q(x, x, y, y)=2 \alpha_{y}^{2}$ for all $y \in \mathbb{M}$, some $a_{y} \in F$.

Proof. Since $x$ is rank one and since strictly regular elements span $M$ by Corollary 6.3 , for all $y \in M, y L_{x, x}=\rho(y) u$ where $\rho$ is a linear functional on $\mathbb{M}$ and $u=z L_{x, x}$ for some fixed, strictly regular $z \in \mathbb{M}$. If $w \perp u,\left\langle w L_{x, x}, y\right\rangle=$ $-\left\langle w, y L_{x, x}\right\rangle=0$ for all $y \in \mathbb{M}$, hence $\rho(w)=0$. This implies $\rho(y)=\beta\langle y, u\rangle$ for all $y \in M$, some $\beta \in F^{*}$, and $\rho(z)=1$ implies $\beta=\langle z, u\rangle^{-1}$. Now $q(x, x, y, y)=\left\langle y, y L_{x, x}\right\rangle=\langle z, u\rangle^{-1}\langle y, u\rangle^{2}$ and $\langle z, u\rangle=\left\langle z, z L_{x, x}\right\rangle=q(z, z, x, x)=$ $\left\langle x, x L_{z, z}\right\rangle=2\langle x, z\rangle^{2}$ give the result.

7.2 Corollary. If $\eta \in G(\mathfrak{M}), \eta$ bas ratio in $\left(F^{*}\right)^{2}$. 
Proof. Let $u_{1}, u_{-1}$ be supplementary strictly regular elements in $\mathbb{M}$ so $q\left(u_{1}, u_{1}, u_{-1}, u_{-1}\right)=2\left\langle u_{-1}, u_{1}\right\rangle^{2}=2$. Since "rank one" is definable entirely in terms of $q\left(q(u, u, x, y)=0\right.$ for all $y \in \mathbb{M}$, all $x \in \mathbb{M}^{\prime}$, $\mathbb{M}^{\prime}$ some $n-1$ dimensional subspace) $u_{1} \eta$ is rank one. By the lemma, $q\left(u_{1} \eta, u_{1} \eta, u_{-1} \eta, u_{-1} \eta\right)=2 a^{2}$ for some $\alpha$. Thus ratio $\eta=a^{2}$ as desired.

If every rank one element in $\mathfrak{M}$ is strictly. regular we obtain a further result.

7.3 Lemma. If rank one implies strictly regular in $\mathbb{M}, G(\mathbb{M}) \subseteq Q(\mathbb{M})$, and if $\eta$ bas ratio $\rho$ in $Q(\mathbb{M})$, it bas ratio $\rho^{2}$ in $G(\mathbb{M})$.

Proof. Let $\eta \in G(\mathbb{M})$ be of ratio $\rho^{2}$. By the argument of the preceding corollary, $u$ strictly regular in $M$ implies $u \eta$ rank one, hence strictly regular. Thus $2\langle u, y\rangle^{2}=q(u, u, y, y)=\rho^{-2} q(u \eta, u \eta, y \eta, y \eta)=2 \rho^{-2}\langle u \eta, y \eta\rangle^{2}$ so $\langle u \eta, y \eta\rangle=$ $\pm \rho\langle u, y\rangle$ for all $y \in \mathbb{M}$. Since for any $y_{i}, i=1,2,\left\langle u \eta,\left(y_{1}+y_{2}\right) \eta\right\rangle= \pm \rho\left\langle u_{0} y_{1}+y_{2}\right\rangle$ $= \pm \rho\left\langle u, y_{1}\right\rangle \pm \rho\left\langle u, y_{2}\right\rangle$, the sign is dependent only on $u$. We can thus partition the set of strictly regular elements into classes $X_{\epsilon}, \epsilon= \pm 1$, depending on the sign of $\rho$ occurring. If $\langle u, v\rangle \neq 0, u, v$ strictly regular, $\epsilon_{u}\langle u, v\rangle=\langle u \eta, v \eta\rangle=$ $-\langle v \eta, u \eta\rangle=-\epsilon_{v}\langle v, u\rangle=\epsilon_{v}\langle u, v\rangle$ so $\epsilon_{u}=\epsilon_{v}$ and $u, v$ are in the same class. In particular, $u_{1}, u_{-1}$ (for some given coordinatization) are in the same class, say $X_{1}$. Moreover, the classes $X_{1}$ and $X_{-1}$ are orthogonal, hence every element of $X_{-1}$ is of form (ii) of Lemma 6.1 . For such an element $\left(0,0, a_{1}, a_{-1}\right)$ we must have also $\left(0,0, a_{1}, 0\right),\left(0,0,0, a_{-1}\right)$ in $X_{-1}$ by an additive argument as above, but the same argument implies $u_{1}+\left(0,0,0, a_{-1}\right) \in X_{-1}$, hence $u_{1} \in X_{-1}$, or $u_{-1}+\left(0,0, a_{1}, 0\right) \in X_{-1}$, hence $u_{-1} \in X_{-1}$, both of which are impossible. It follows that $a_{-1}=a_{1}=0$ and $X_{-1}=\{0\}$. Thus the sign of $\rho$ is constant for all strictly regular $u$, all $y \in \mathbb{M}$. Since the strictly regular elements span $\mathbb{I}$ the result follows.

In particular, Corollary 6.2 implies that the result of the lemma holds for $M=M(\Im), ~ \Im$ the Jordan algebra of an admissible cubic form, a result analogous to that in [3, Lemma 1]. The result is false, however, when $\mathcal{S}$ is Jordan algebra of a quadratic form since in this case $q(x)=12 B(x)^{2}$ where $B(x)$ is a quadratic form. It is easy to show that there is $T \in \operatorname{Hom}(\mathbb{M}, \mathbb{M})$ such that $B(x T)=\lambda \boldsymbol{B}(x)$ for all $x \in \mathbb{M}$ but $\langle x T, y T\rangle \neq \pm \lambda\langle x, y\rangle$.

It is clear that the results $6.6,6.7,7.2$ and 7.3 remain valid when the transformations in question are replaced by their semilinear analogues. The same will be seen to be true of Lemma 7.5 , though for brevity we restrict our attention to the linear case.

We define now a particularly simple type of transformation from $\mathbb{M}\left(\mathfrak{\Im}_{1}\right)$ to $\mathfrak{M}\left(\mathfrak{\Im}_{2}\right)$ which preserves forms up to scalar multiples. Let $\mathfrak{I}_{1}, \mathfrak{I}_{2}$ be Jordan algebras as in $\S 1, N_{1}, N_{2}$ the associated cubic forms and $\eta \in \operatorname{Hom}\left(\mathfrak{I}_{1}, \mathfrak{J}_{2}\right)$ 
such that $N_{2}(a \eta)=\rho N_{1}(a)$ for all $a \in \mathfrak{J}_{1}$, some $\rho \in F^{*}, \eta$ bijective. Let $\pi$ be a permutation on $\{1,-1\}$ and $\lambda \in F^{*}$. Define $\eta^{*} \in \operatorname{Hom}\left(\mathfrak{S}_{2}, \mathfrak{S}_{1}\right)$ by $T_{2}(a, b \eta)=$ $T_{1}\left(a \eta^{*}, b\right), T_{i}(-,-)$ denoting the trace form on $\mathfrak{S}_{i}$. Clearly $T_{2}\left(a \eta^{*-1}, b \eta\right)=$ $T_{1}(a, b)$ for all $a, b \in \Im_{1}$. We have also $(a \eta)^{\# 2}=\rho a^{\# 1} \eta^{*^{-1}},\left(a \eta^{*^{-1}}\right)^{\# 2}=\rho^{-1} a^{\# 1} \eta$ and $N_{2}\left(a \eta^{*-1}\right)=\rho^{-1} N_{1}(a)$ since these are trivial if $\mathfrak{J}_{i}$ are algebras of quadratic forms and are 1.21 and 1.22 of [4] if $\mathfrak{S}_{i}$ are algebras of cubic forms.

One can verify easily that the linear mapping defined by

$$
\begin{aligned}
\eta_{\pi}^{\lambda}: u_{1} & \rightarrow \lambda^{-1} \rho^{-1} u_{1 \pi}, \quad u_{-1} \rightarrow \lambda^{2} \rho u_{(-1) \pi}, \\
a_{1} & \rightarrow \lambda(a \eta)_{1 \pi}, \quad a_{-1} \rightarrow\left(a \eta^{*-1}\right)_{(-1) \pi}, \quad \lambda \in F^{*},
\end{aligned}
$$

satisfies $q_{2}\left(x \eta_{\pi}^{\lambda}\right)=\lambda^{2} q_{1}(x),\left\langle x \eta_{\pi}^{\lambda}, y \eta_{\pi}^{\lambda}\right)_{2}=(1 \pi) \lambda(x, y\rangle_{1}$ for all $x, y \in \mathscr{M}\left(\mathfrak{J}_{1}\right)$.

This gives immediately the converse to Theorem $\} .8$.

7.4 Theorem. If there is bijective $\eta \in \operatorname{Hom}(\Im, \Omega)$ sucb that $N_{\Re}(a \eta)=$ $\rho N_{\mathcal{Y}}(a)$ for all $a \in \Im$, some $\rho \in F^{*}$, then $\mathbb{M}(\Im) \cong \mathbb{M}(\Omega)$.

Proof. Take $\eta_{I}^{1}$ and use Lemma 6.6.

We also have half of the characterization of a class of elements of $G(\mathbb{R}(\Im))$, namely

7.5 Lemma. Let $u_{\epsilon}, \epsilon= \pm 1$, be the usual supplementary, strictly regular elements in $\mathbb{M}(\mathfrak{S})$. $\phi \in G(\mathbb{M})$ leaves invariant $F u_{1}+F u_{-1}$ if and only if there is $\eta \in \operatorname{Hom}(\Im, \Im)$ sucb that $N(a \eta)=\rho N(a)$ for all $a \in \mathfrak{J}$, a permutation $\pi$ of $\{1,-1\}$ and $\lambda \in F^{*}$ sucb that $\phi=\eta_{\pi}^{\lambda}$.

Proof. Clearly $\eta_{\pi}^{\lambda}$ is of the desired type. Suppose $\phi \in G(\mathbb{M})$ leaves $F u_{1}+F u_{-1}$ invariant. $\phi$ is nonsingular since otherwise $q(x, y, z, w)=\langle x, y z w\rangle$ $=0$ for some $x$, all $y, z, w \in \mathbb{M}$, which implies $(F x)^{\perp}$ is an ideal in the simple algebra $M$. From Corollary 3.7 we have, since $\phi \in G(\mathbb{M}), u_{\epsilon} \phi=\rho_{\epsilon} u_{\epsilon \pi}, \pi$ a permutation of $\{1,-1\}$. Replacing $\phi$ by $\phi_{1}=\phi\left(I_{\pi}^{1}\right)^{-1}$ we may assume $u_{\epsilon} \phi_{1}=$ $\rho_{\epsilon} u_{\epsilon}$. This implies $q\left(x \phi_{1}\right)=\left(\rho_{1} \rho_{-1}\right)^{2} q(x),\left\langle x \phi_{1}, y \phi_{1}\right\rangle=\rho_{1} \rho_{-1}\langle x, y\rangle$. For $\lambda=\rho_{1} \rho_{-1}$ we have $\phi_{2}=\phi_{1}\left(I_{l}^{\lambda}\right)^{-1}$ is an automorphism by Corollary 6.7. Thus $\phi_{2}$ maps Peirce spaces onto themselves. Denoting by $a_{1}$ (resp. $a_{-1}$ ) the element $(0,0, a, 0)$ (resp. $(0,0,0, a)), a \in \Im$, we define $\eta_{\epsilon} \in \operatorname{Hom}(\mathfrak{J}, \mathfrak{J})$ by $a_{\epsilon} \phi_{2}=\left(a \eta_{\epsilon \epsilon}\right.$. Again writing $u_{\epsilon} \phi_{2}=\rho_{\epsilon} u_{\epsilon}$ and noting $\rho_{1} \rho_{-1}=1$ we have $\rho_{-1}=\rho_{1}^{-1}$. Applying $\phi_{2}$ to $u_{1}+a_{1}$ and evaluating $q(-)$ yields $N(a)=\rho_{1} N\left(a \eta_{1}\right)$ and similarly $N(a)=\rho_{-1} N\left(a \eta_{-1}\right)$. Since $\left\langle a_{1} \phi_{2}, b_{-1} \phi_{2}\right\rangle=\left\langle a_{1}, b_{-1}\right\rangle$ we have $T\left(a \eta_{1}, b \eta_{-1}\right)=T(a, b)$, hence $\eta_{-1}=\eta_{1}^{*}-1$. It follows immediately that $\phi_{2}=\eta_{I}^{1}$ where $\eta=\eta_{1}$, hence $\phi=\eta_{I}^{1} I_{I}^{\lambda} I_{\pi}^{1}=\eta_{\pi}^{\lambda}$ as desired.

We now have

7.6 Theorem. Let $\left(u_{1}, u_{-1}\right),\left(v_{1}, v_{-1}\right)$ be supplementary pairs of strictly 
regular elements in $\mathbb{M}$, $M$ simple. Then there is $\phi \in$ Aut $\mathbb{M}$ sucb that $u_{\epsilon} \phi=$ $\rho_{\epsilon} v_{\epsilon}, \rho_{\epsilon} \in F^{*}$.

Proof. Replacing the pairs $\left(u_{\epsilon}\right),\left(v_{\epsilon}\right)$ by suitable scalar multiples $\left(\tilde{u}_{\epsilon}\right)$, $\left(\tilde{v}_{\epsilon}\right)$ we can coordinatize $\mathbb{M}$ to obtain $\mathfrak{M} \cong \mathbb{M}(\Im), \mathfrak{M} \cong \mathbb{M}(\Omega)$ where $\mathfrak{J}$, $\Omega$ are Jordan algebras with cubic forms $(1 / 12) N_{\tilde{u}_{1}, \tilde{u}_{-1}},(1 / 12) N_{\widetilde{v}_{1}, \tilde{v}_{-1}}$ respectively. By Lemma 6.5 there is $\eta: \breve{S} \rightarrow \Re$ such that $N_{\mathcal{R}}(a \eta)=\rho N_{\mathcal{J}}(a)$ for all $a \in \mathfrak{S}$, some $\rho \in F^{*}$. The mapping $\phi=\eta_{I}^{1}$ is thus an automorphism with the desired properties.

7.7 Corollary. Aut $M$ acts transitively on the set of strictly regular elements (taken projectively).

Proof. The result follows immediately from the fact that every strictly regular element can be embedded in a supplementary pair (see proof of Theorem 3.3).

7.8 Corollary. $G(\Re)$ acts transitively on the set of strictly regular elements.

Proof. Immediate from Corollary 7.7 and the fact that $\phi \in$ Aut $\Re, \rho \in F^{*}$ implies $\rho \phi \in G(\mathbb{M})$.

We denote by $R($ ( ) $)$ the subgroup of $F^{*}$ of all $\rho$ such that there is $\eta \epsilon$ $\operatorname{Hom}(\mathfrak{S}, \mathfrak{J})$ with $N(a \eta)=\rho N(a)$ for all $a \in \mathfrak{J}$. We then have

7.9 Corollary. The number of conjugacy classes of supplementary pairs of strictly regular elements in $\mathfrak{M}(\mathfrak{S})$ under the action of Aut $\mathbb{M}(\mathfrak{S})$ is the index of $R\left(\right.$ (s) in $F^{*}$.

Proof. If $u_{1}, u_{-1}$ are the canonical strictly regular elements in $\mathbb{M}(\Im)$, Theorem 7.6 implies that any supplementary pair is conjugate to a pair $\left(\lambda u_{1}, \lambda^{-1} u_{-1}\right)$. It thus suffices to determine the number of conjugacy classes among these pairs. Suppose $\phi \in A u t M(\Im), u_{\epsilon} \phi=\rho_{\epsilon} u_{\epsilon}$. Lemma 7.5 implies $\phi=\eta_{I}^{\lambda}$ and the fact that ratio $\phi=1$ (Corollary 6.7) implies $\lambda=1$, hence $\rho_{1} \in R(\Im)$. If $u_{\epsilon} \phi=\rho_{\epsilon} u_{-\epsilon}, u_{1} \phi I_{(1,-1)}^{-1}=\rho_{-1} u_{1}$ so $\rho_{-1} \in R(\Im)$. Since $\rho_{1}=$

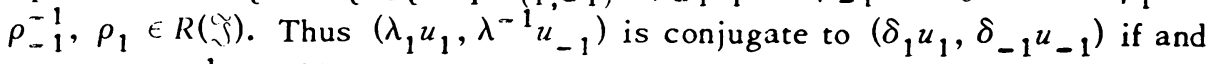
only if $\lambda_{1} \delta_{1}^{-1} \in R(\Im)$.

Since the results of $\S 3$ show that elements $x$ with $q(x)=12$ are uniquely expressible as sums of supplementary pairs of strictly regular elements, Corollary 7.9 could also be phrased in terms of conjugacy classes of elements with $q(x)=12$. In the particular case $M=M(\Im), \zeta$ reduced, exceptional simple, the fact that $R(\Im)=F^{*}$ gives as a special case the result of Brown [1].

7.10 Theorem. If $\breve{\zeta}$ is reduced, exceptional simple, Aut $\mathbb{M}(\Im)$ acts transitively on the set of $x \in \mathbb{M}(\widetilde{J})$ with $q(x)=12$. 
8. Simple FTS's over special fields. In this section we apply the techniques

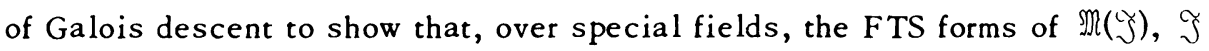
exceptional central simple Jordan, are always reduced.

Using the usual terminology of Galois descent, if $F \subseteq K$ are fields and $\tilde{\mathscr{M}}$ is a FTS over $K$, and $F$-form of $\tilde{M}$ is an $F$-subspace of $\tilde{M}$, closed under the product of $\tilde{\mathfrak{M}}$, such that $K \mathbb{M}=\tilde{M}$, $\operatorname{dim}_{F} \mathfrak{M}=\operatorname{dim}_{K} \tilde{\mathfrak{M}}$. If $K$ is finite Galois over $F$ with group $G$ it is well known [8] that the $F$-forms of $K$ are in one-one correspondence with the homomorphisms $\eta: G \rightarrow$ Saut $\tilde{\mathscr{M}}$, Saut $\tilde{\mathscr{M}}$ the group of semilinear automorphisms, where $\eta(s)$ is $s$-semilinear. The correspondence is given simply by $\eta \leftrightarrow \mathbb{M}_{\eta}=\{x \in \tilde{\mathbb{M}} \mid x \eta(s)=x \quad \forall s \in G\}$.

If $\stackrel{M}{M}$ is an $F$-form of $\tilde{M}, x \in \mathbb{M}$, A3 with $x=y$ yields $(x x x) x x=q(x) x$, hence $q(x) \in F$ for all $x \in \mathbb{M}$. This implies $\langle y, x x x\rangle \in F$ for all $y \in \mathbb{M}$ and that $q$ induces a nontrivial quartic form on $\mathbb{M}$. If $q(x) \neq 0, x$ and $x x x$ are linearly independent in $\widetilde{M}$, hence in $\mathbb{M}$, so $\mathrm{A} 3$ implies $\langle y, x\rangle \in F$ for all $y \in \mathbb{M}$. A density argument then shows $\langle\ldots, \ldots\rangle$ induces a skew form on $\mathbb{M}$ and $\mathbb{M}$ is a FTS.

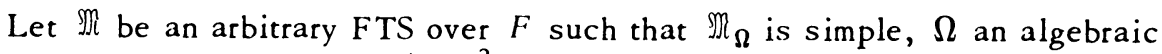
closure of $F$. Suppose $\beta \in F^{*} \backslash F^{*^{2}}$ and $x \in \mathbb{M}$ with $q(x)=\beta$. Then $\mathbb{M}_{K}$ is reduced, simple for $K=F(\bar{\beta}), \bar{\beta}=(\beta / 12)^{1 / 2}$, by Corollary 3.4. In fact, Lemma 3.6 shows that in $M_{K}, x$ is uniquely expressible as a sum of strictly regular elements $u_{1}, u_{-1}$. By the results of $\oint_{5}$, for suitable $\delta_{i} \in K^{*}$, we can coordina= tize $\mathbb{M}_{K}$ as $\mathfrak{M}(\breve{\jmath})$ relative to the pair $\tilde{u}_{1}:=\delta_{1} u_{1}, \tilde{u}_{-1}=\delta_{2} u_{-1}$. The Galois descent characterization of forms of $\mathbb{M}_{K}$ shows that $\mathbb{M}$ is the fixed point set of some semiautomorphism $\phi$ of period two on $\mathbb{M}_{K}$. Since $x \phi=x, u_{\epsilon} \phi=\rho_{\epsilon} u_{ \pm \epsilon}$ so, by the semilinear analogue to Lemma 7.5, $\phi-\eta_{\pi}^{\lambda}$ for some permutation $\pi$ of $\{1, \cdots 1\}$, some $\lambda \in K^{*}$, and some semilinear map $\eta: \Im \rightarrow \Im$ such that $N(a \eta)-$ $\rho N(a)^{s}$ for all $a \in Y, s$ denoting the nontrivial $F$-automorphism of $K$. Since $\phi$ is a semiautomorphism we must also have $\lambda .1 \pi$ by Corollary 6.7 and, since $\phi^{2} . I$ we have either $\eta^{2}-I$ if $\pi=I$ or $\eta^{*}-\cdots \eta$ if $\pi=(1, \ldots 1)$.

If $\pi=I, \tilde{u}_{1} \phi-\rho_{1} \tilde{u}_{1}$ so $\phi^{2}:=I$ implies $\rho_{1} \rho_{1}^{s}=1$ and thus $\rho_{1}=\gamma^{-1} \gamma^{s}$ for some $\gamma \in K^{*}$. $\phi$ thus fixes $\gamma^{s} \widetilde{u}_{1}$ which is strictly regular, hence $\mathbb{M}$ contains a strictly regular element and is reduced.

If $\pi ; l$ we have for $\mathbb{M}_{K} \ldots M\left(M_{i}\right)$

8.1 I.emma. $M$ is reduced if and only if there is $a \in \xi, N(a) \neq 0$ and $\delta \in K^{*}$ such tbat $a \eta \quad \delta a^{\#}$.

Proof. If $M$ is reduced, $M$ ? is spanned by strictly regular elements by Corollary 6.3. Thus there is strictly regular $y=(\alpha, \beta, a, b) \in M((\breve{s})$ with $\alpha \neq 0$ such that $y \phi \cdots y$. Since $\phi$ interchanges $\hat{u}_{1}^{\prime}$ and $\hat{u}_{-1}^{\prime}$ this implies $\beta \neq 0$ and Lemma 6.1 thus implies $b \ldots \delta a^{\sharp}, N(a) \neq 0$ which establishes the necessity of the condition. 
Conversely if $a \eta=\delta a^{\#}$ (note that this immediately implies $\mathcal{S}$ is not the algebra of a quadratic form $),\left(\delta^{s}\right)^{-1} a=a^{\#} \eta^{-1}=-a^{\#} \eta^{*-1}=-\rho^{-1}(a \eta)^{\#}=$ $-\rho^{-1} \delta^{2} a^{\#}=-\rho^{-1} \delta^{2} N(a) a$ (using conditions relating $\eta$ and \# preceding (15), \$7). Thus $\rho=-\delta^{s} \delta^{2} N(a)$ and it is easy to verify that $\phi$ fixes the strictly regular element $y=\left(\rho \delta^{-s}, \delta^{-1},-a, \delta a^{\sharp}\right)$ since $\eta^{*}=-\eta$ implies $\rho^{s}=-\rho$.

An immediate consequence of the lemma is that, for suitably selected fields $F$, there exist nonreduced, simple FTS's. The simplest example involves $K=F(\sqrt{\mu})$, a quadratic extension of $F$ with nontrivial automorphism $\sigma$. Let $\mathfrak{S}$ be Jordan algebra of a quadratic form over $F$ and let $\eta=\sqrt{\mu}(I \oplus \sigma)$ act on $\mathfrak{\Im}_{K}$. The semilinear map $\phi=\eta_{(1,-1)}^{\lambda}, \lambda=-1, \rho=\sqrt{\mu}$, is a semiautomorphism of $\mathbb{M}\left(\Im_{K}\right)$ of order 2 which fixes a simple $F$-form $M$ which cannot be reduced since $\eta$ is nonsingular and $a^{\#}=0$ for all $a \in \mathfrak{J}_{K}$. A less trivial example occurs if $F$ is taken to be the rational function field $Q\left(x_{1}, x_{2}, x_{3}, x_{4}\right), K=F(\sqrt{-1})$. The semilinear map $C(s)$ given in [6] acts on a split exceptional algebra $\mathfrak{J}$ over $K$ and has property $a C(s) \neq \delta(a \times a)$ for all $a \in \Im, N(a) \neq 0$. Thus the semiautomorphism $\eta_{(1,-1)}^{\lambda}, \lambda=-1, \eta=\sqrt{-1} C(s)$ defines a nonreduced $F$-form of $\mathbb{M}(\Im)$. We have finally

8.2 Theorem. Let $\mathbb{M}$ be an F-form of a FTS M( $(\mathfrak{S})$, $\mathfrak{S}$ exceptional simple. Then $M$ is reduced if $F$ is finite, real, p-adic, or algebraically closed.

Proof. The algebraically closed case is just Corollary 3.5. In the remaining cases pick $x \in \mathfrak{M}$ with $q(x)=\beta \neq 0$. If $\beta / 12$ is a square, $\mathbb{M}$ is reduced by Corollary 3.4. We thus assume $K=F(\bar{\beta}), \bar{\beta}=(\beta / 12)^{1 / 2}$, is a quadratic extension of $F$ such that $\mathbb{M}_{K}=\mathfrak{M}(\overline{\mathfrak{J}}), \overline{\mathfrak{J}}$ an exceptional Jordan algebra, and such that $x=u_{1}+u_{-1}, \widetilde{u}_{\epsilon}$ the canonical strictly regular elements (see above remarks). $\mathbb{M}$ is thus the fixed point set of a semiautomorphism $\eta_{\pi}^{\lambda}$ as above. If $\pi$ is the identity, $\mathfrak{M}$ is reduced. Thus we assume $\pi=(1,-1), \lambda=-1, \eta^{*}=-\eta$. It suffices to show that, for the given fields, such an $\eta$ always satisfied the conditions of Lemma 8.1. For this we replace $\eta$ by $\bar{\beta} \eta=\bar{\eta}$ to obtain $\bar{\eta}^{*}=\bar{\eta}$ and observe that precisely this result is proved in [6, Lemma 3 and preceding statements and proof of Theorem 5].

Note. The theorem is also true if $F$ is an algebraic number field. A proof of this fact will be incorporated in a forthcoming paper by the author on classification of Lie algebras of type $E_{7}$.

\section{BIBLIOGRAPHY}

1. R. B. Brown, Groups of type $E_{7}$, J. Reine Angew. Math. 236 (1969), 79-102. MR $40 \# 1439$.

2. J. R. Faulkner, A construction of Lie algebras from a class of ternary algebras, Trans. Amer. Math. Soc. 155 (1971), 397-408. 
3. J. R. Faulkner, A geometry for $E_{7}$, Trans. Amer. Math. Soc. 167 (1972), 49-58.

4. - Octonion planes defined by quadratic Jordan algebras, Mem. Amer. Math. Soc. No. 104 (1970). MR $42 \# 6063$.

5. J. R. Faulkner and J. C. Ferrar, On the structure of symplectic ternary algebras, Indag. Math. 34 (1962), 247-256.

6. J. C. Ferrar, Lie algebras of type $E_{6}$, J. Algebra 13 (1969), 57-72. MR 41 \#8480.

7. H. Freudenthal, Beziehungen der $E_{7}$ und $E_{8}$ zur Oktavenebene, Nederl. Akad. Wetensch. Proc. Ser. A 57 = Indag. Math. 16 (1954), 218-230. MR 16, 108.

8. N. Jacobson, Forms of algebras, Some Recent Advances in the Basic Sciences, vol. 1 (Proc. Ann. Sci. Conf., Belfer Grad. School Sci., Yeshiva Univ., New York, 19621964), Belfer Graduate School of Science, Yeshiva Univ., New York, 1966, pp. 41-71. MR $35 \# 5477$.

9. K. Meyberg, Eine Theorie der Freudenthalschen Tripelsysteme. I, II, Nederl. Akad. Wetensch. Proc. Ser. A 71 = Indag. Math. 30 (1968), 162-174, 175-190. MR 37 $\# 1429$.

10. K. McCrimmon, The Freudenthal-Springer-Tits constructions of exceptional Jordan algebras, Trans. Amer. Math. Soc. 139 (1969), 495-510. MR 39 \#276.

11. T. A. Springer, Characterization of a class of cubic forms, Nederl. Akad. Wetensch. Proc. Ser. A 65 = Indag. Math. 24 (1962), 259-265. MR 25 \#2104.

12. - On a class of Jordan algebras, Nederl. Akad. Wetensch. Proc. Ser. A 62 = Indag. Math. 21 (1959), 254-264. MR 22 \#1607.

DEPARTMENT OF MATHEMATICS, OHIO STATE UNIVERSITY, COLUMBUS, OHIO 43210 